\title{
Performance Limits of the Three MEMS Inertial Energy Generator Transduction Types
}

\author{
P. D. Mitcheson ${ }^{1}$, E. K. Reilly ${ }^{2}$, T. Toh ${ }^{1}$, P. K. Wright ${ }^{2}$ and E. M. Yeatman ${ }^{1}$ \\ ${ }^{1}$ Department of Electrical and Electronic Engineering, Imperial College London \\ ${ }^{2}$ Department of Mechanical Engineering, University of California Berkeley
}

\begin{abstract}
In this paper, the trends from the last 10 years of inertial micro-generator literature are investigated and it is shown that, although current generator designs are still operating well below their maximum power, there has been significant improvement with time. Whilst no clear conclusions could be drawn from reported fabricated devices with respect to preferred transducer technology, this paper presents operating charts for inertial micro-generators which identify optimal operating modes for different frequencies and normalized generator sizes, and allows comparison of the different transduction mechanisms as these parameters vary. It is shown that piezoelectric generators have a wider operating range at low frequency than electromagnetic generators, but as generator dimensions increase, the frequency to which piezoelectric transducers outperform electromagnetic transducers decreases.
\end{abstract}

\section{Keywords: Energy-harvesting, piezo-electric, ultra low-power}

\section{1 - INTRODUCTION}

Motion and vibration are attractive sources for microengineered energy scavenging generators $[1,2]$. The most universal motion scavengers are of the inertial type, i.e. having a proof mass suspended within a frame, and energy extracted by a transducer that damps the motion of the proof mass within the frame. These devices have the advantage that they can function simply by being attached to a source of motion at a single point, rather than relying on the relative motion of different parts of the "host" structure. Thus they are also well suited to miniaturisation.

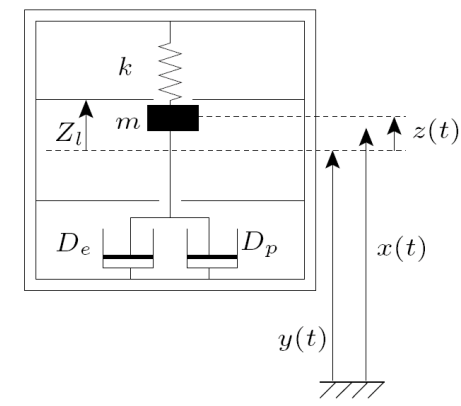

Figure 1 - Schematic construction of inertial generators.

The basic operating principle of inertial micro-generators is illustrated in Fig. 1. The fundamental parameters limiting the generator output are its proof mass $m$ and maximum internal displacement $Z_{l}$, and the source motion amplitude $Y_{0}$ and frequency $\omega[3]$. From these we can derive the maximum power from basic principles. If we assume harmonic source motion, the maximum acceleration $a_{\max }$ is $\omega^{2} Y_{0}$. The maximum damping force by which energy can be extracted is equal to the inertial force on the proof mass, $m a_{\max }$ (if greater, the mass will not move relative to the frame). If energy is extracted in both directions, and the internal motion amplitude $Z_{\mathrm{o}}=Z_{l}$, (giving the maximum travel range of $2 Z_{l}$ ) we derive a total energy per cycle of $4 Z_{l} m a_{\max }=4 Z_{l} m \omega^{2} Y_{0}$. To convert this to power we simply divide by the excitation period $2 \pi / \omega$, giving:

$$
P_{\max }=2 Y_{0} Z_{l} \omega^{3} m / \pi
$$

We can then define a normalised power $P_{n}=P / P_{\max }$ as a measure of how close the performance of a specific device comes to the optimum level. We have calculated $P_{n}$ for measurements on inertial energy scavengers reported in the literature [1, 4-27] and the resulting values are plotted in Fig. 2 as a function of year of publication. An upwards trend can clearly be seen, although the best values are still below $20 \%$ of the optimum. Although $P_{n}$ should not drop with volume, since it is normalised to device size, the same data plotted against device volume (Fig. 3) show that typically the best $P_{n}$ values have been achieved for larger devices. This is likely an indication of the technological difficulties encountered at smaller size scales, for example the greater difficulty in achieving high magnetic flux gradients. Finally, we plot the normalised power vs. frequency (Fig. 4), and a downwards trend is clearly seen.

Several transduction mechanisms can be used for inertial micro-generators, namely electromagnetic [14], electrostatic [28] and piezoelectric [29]. The transduction type is also indicated in Figs. 2 - 4, but no clear trends can be seen regarding their relative merits. To obtain some general guidance on the practical limitations of specific transduction methods, we have examined the key issue of the damping

${ }^{*}$ Contact author: Tel. +44-20-7594-6284, email: paul.mitcheson@imperial.ac.uk 
levels that can be achieved. We consider only mechanically resonant devices operating at the resonant frequency $\omega_{n}$, as this covers most reported examples.

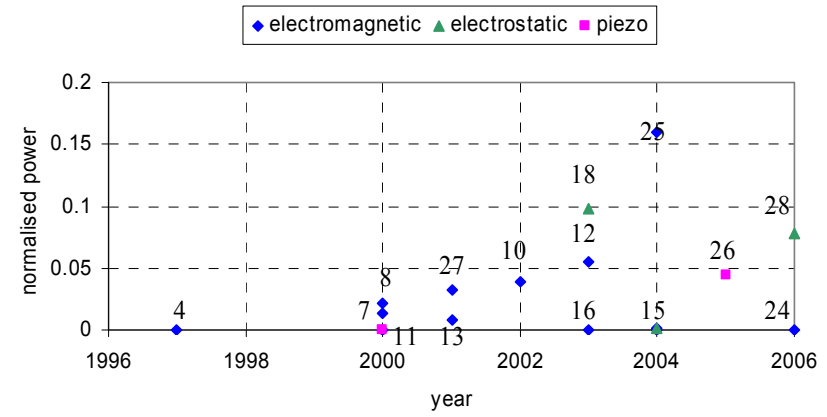

Figure 2 - Normalised measured power $P_{n}$ vs. year of publication. Numbers show which reference the data was taken from

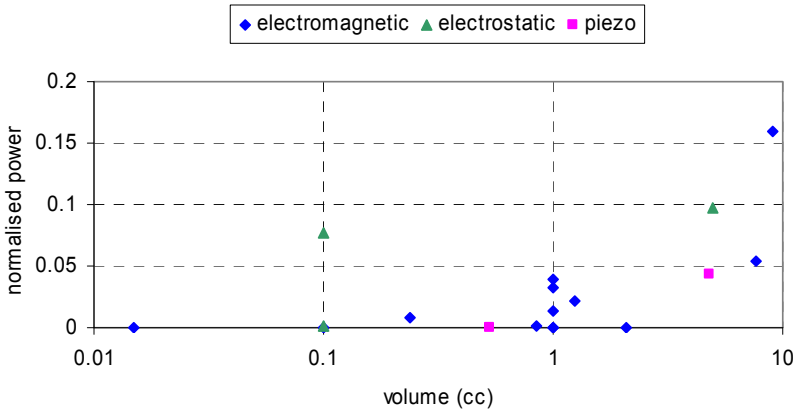

Figure 3 - Normalized measured power $P_{n}$ vs. device volume.

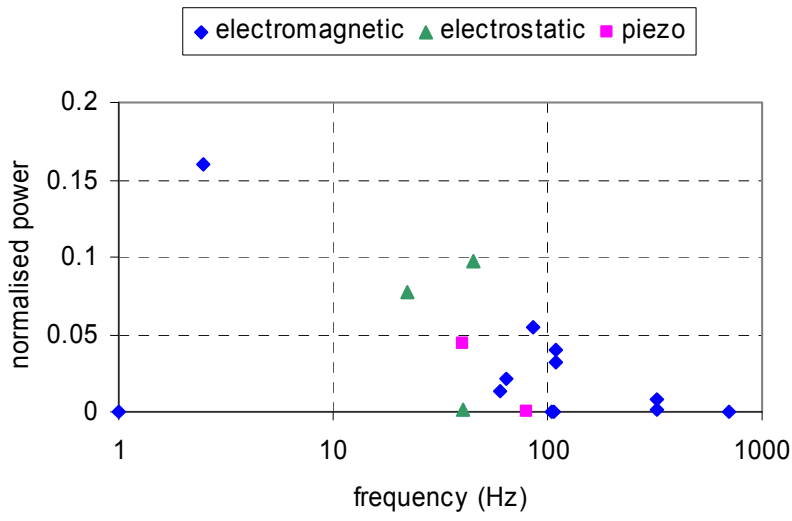

Figure 4 - Normalised measured power $P_{n}$ vs. operating frequency.

\section{2 - SCALING ANALYSIS OF TRANSDUCER TYPES}

In an ideal case, the parasitic damping would be zero, and maximum power is then obtained by setting the electrical damping factor $\zeta_{e}$ to the level that allows the mass to move over the entire internal range, but without hitting the endstops, i.e. $Z_{0}=Z_{l}$ [3]. However, in some cases the maximum damping force of the transducer is insufficient to achieve this, and thus the device cannot operate in a resonant mode. In cases where the parasitic damping factor $\zeta_{p}$ is not negligible, maximum power will inevitably be reduced, but the optimum $\zeta_{e}$ will still be that which gives $Z_{0}=Z_{l}$, unless this requires $\zeta_{e}<\zeta_{p}$, in which case $\zeta_{e}=\zeta_{p}$ should be chosen if possible.
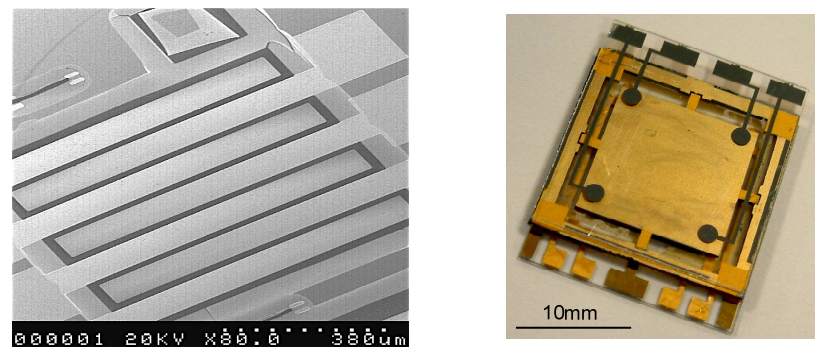

Figure 5 - Prototype MEMS inertial scavengers: piezoelectric (left) and electrostatic (right).

In general, the damping factor is related to the resonant quality factor $Q$ by $Q=1 / 2 \zeta$. Since the damping will have parasitic and electrical (transducer) components, we introduce the quantities $Q_{p}=1 / 2 \zeta_{p}$ and $Q_{e}=1 / 2 \zeta_{e}$. Note that the combined $Q$ is given by $1 / Q=1 / Q_{p}+1 / Q_{e}$. Furthermore, for a resonant system in which the damping force is proportional to the relative mass-frame velocity, i.e. $F=-D d z / d t, D=2 m \omega_{n} \zeta$. To perfectly damp the system we require $Q=Z_{l} / Y_{0}$, so that $D=m \omega_{n} /\left(Z_{l} / Y_{o}\right)$.

\section{1 - Electromagnetic Damping}

An electromagnetic damper can be implemented as a coil moving across the boundary of a region of magnetic flux density B. If we assume that the induced voltage is limited mainly by the resistive load $\mathrm{R}$, rather than the coil's inductance, then in this case the electrical damping coefficient is given by $D_{e}=(N B l)^{2} / R$ [3], where $N$ is the number of coil turns and $l$ is the length of the border of the flux region cutting across the coil. The load $\mathrm{R}$ will consist of the coil resistance $R_{i}$ (which is a parasitic component) and the energy extracting load $R_{L}$ in series. The former determines the maximum $D_{e}$, and thus the minimum $Q_{e}$; however, unless $R_{L}$ is substantially greater than $R_{i}$ most of the electrical power will be wasted. Instead we assume that a useful device has at least $R_{L}=10 R_{i}$. This sets the minimum achievable $Q_{e}$ as:

$$
Q_{e}(\min )=\frac{10 m R_{i}}{(N B l)^{2}} \omega_{n}
$$

If this quantity is greater than $Z_{l} / Y_{0}$, it will not be possible to achieve harmonic motion by electrical damping alone, although it may be possible if significant parasitic mechanical damping is present. A key implication of (2) is that the range of achievable $Q_{e}$ is reducing with increasing frequency. 


\section{2 - Piezoelectric Damping}

A similar quantity can be derived for the piezoelectric case. We start with the constituent equations for the piezoelectric cell [30]:

$$
\begin{aligned}
& F_{p}=K_{P E} z+\alpha V \\
& I=\alpha \dot{z}-C_{0} \dot{V}
\end{aligned}
$$

Here $F_{p}$ is the force produced by the piezoelectric material, $V$ is the voltage between the terminals, $I$ is the current through the terminals, $K_{P E}$ is the short circuit stiffness, z the relative displacement, $C_{0}$ is the capacitance of the piezoelectric element and $\alpha=e_{33} A / t$, with $A$ and $t$ the cross-sectional area and thickness of the piezo element, and $e_{33}$ the piezoelectric coefficient. Note that $e_{33}$ applies to the case where the voltage and strain are colinear; many implementations, such as thin piezo films on cantilevers, have strain and electric field on orthogonal axes, and an off-diagonal element of the piezoelectric tensor then applies in place of $e_{33}$. The force developed by the piezoelectric cell with a resistive load $R$ connected can be found from these equations, in the Laplace domain, as:

$$
F(s)=Z(s)\left(K_{P E}+\frac{s \alpha^{2} R}{1+s C_{0} R}\right)
$$

The force developed by the piezoelectric material is therefore a constant spring force plus a force which acts as a first order high pass filter. The frequency response, $F_{H P}(j \omega)$, of the high pass filter term is:

$$
F_{H P}(j \omega)=Z(j \omega)\left(\frac{j \omega \alpha^{2} R}{1+j \omega C_{0} R}\right)
$$

which can be written as:

$$
F_{H P}(j \omega)=Z(j \omega)\left(\frac{j \omega \alpha^{2} R}{1+\left(\omega C_{0} R\right)^{2}}+\frac{\omega^{2} \alpha^{2} C_{0} R^{2}}{1+\left(\omega C_{0} R\right)^{2}}\right)(7)
$$

These terms are therefore a frequency dependent damping term (proportional to $j \omega Z(j \omega)$ ) and a frequency dependent spring constant term (proportional to $Z(j \omega)$ ). The resistance can be altered to maximise the available damping force, $F_{D}(j \omega)$, i.e. when:

$$
\frac{d F_{D}(j \omega)}{d R}=0
$$

$$
R=\frac{1}{\omega C_{0}}
$$

Thus,

$$
D_{\max }=\frac{\alpha^{2}}{2 \omega C_{0}}
$$

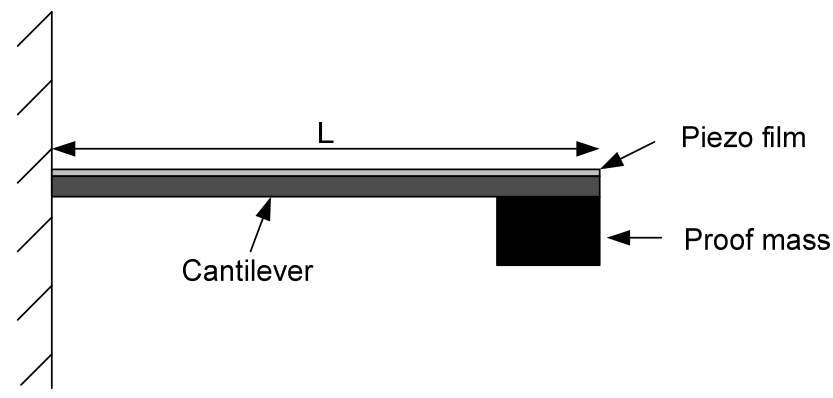

Figure 6 Piezoelectric film on cantilever beam

In practice, since piezo elements can only achieve very small direct displacements, devices must incorporate some leverage mechanism (of ratio $r$ ). A typical example is the cantilever structure shown in Fig. 6. If we approximate this as a thin film of piezoelectric material on a structural cantilever of thickness $h$, then it is straightforward to show that the proof mass motion $\Delta z$ is greater than the piezo film extension (or compression) by a factor $L / h$.

The leverage will transform the damping coefficient (i.e. the force to velocity ratio) by a factor of $r^{2}$, in a manner equivalent to an electrical transformer of turns ratio $r$. Thus we obtain:

$$
Q_{e}(\min )=\frac{2 m C_{o} r^{2}}{\alpha^{2}} \omega_{n}^{2}
$$

Since the other parameters in this expression have little or no frequency dependence, the minimum $Q_{e}$ is proportional to frequency squared. Thus the operating range for which optimum power can be achieved becomes rapidly diminished with increasing frequency; more rapidly so than in the electromagnetic case considered above.

\section{3 - Electrostatic Damping}

For the case of the electrostatic damper, the damping is non-linear but a closed form solution to the minimum available $Q$ factor exists. This is given in [3] as:

Which is when: 
$Q_{e}(\min )=\omega_{c}^{2}\left[\frac{1}{\left(1-\omega_{c}^{2}\right)^{2}}-\left(\frac{F \max }{m Y_{0} \omega^{2} \omega_{c}} U\right)^{2}\right]^{\frac{1}{2}}$

where:

$$
U=\frac{\sin \left(\frac{\pi}{\omega_{c}}\right)}{1+\cos \left(\frac{\pi}{\omega_{c}}\right)}
$$

There are two different methods of operating an electrostatic transducer to achieve Coulomb (fixed force) damping, which are gap-closing constant charge operation, and sliding plate constant voltage operation. The most common type on the microscale is the latter, in the form of a comb drive variable capacitor on a folded suspension. For this type of transducer the parasitic damping force can be approximated as [31]:

$$
F_{p}(\dot{z})=\frac{2 N_{g} t \mu_{a i r} x_{o}}{g} \dot{z}
$$

Here $N_{g}$ is the number of gaps in the comb drive, $t$ is the thickness of the structure, $\mu_{\text {air }}$ is the viscosity of air, $g$ is the gap width, $x_{o}$ is the initial gap overlap, and $z$ is the overlap movement. The electrical damping force is given by:

$$
F_{e}(z)=\frac{Q_{c}^{2}}{2 C_{o}} \frac{1}{\left(z+x_{o}\right)^{2}}
$$

where $Q_{c}$ is the initial charge on the capacitor, and $\mathrm{C}_{\mathrm{o}}$ is the capacitance per given length as given by:

$$
C_{o}=\frac{N_{g} \varepsilon_{0} t}{g}
$$

It can be seen that the electrical damping force is not proportional to velocity, as is required for harmonic oscillation. Thus the motion will be inherently nonlinear. Furthermore, it is a property of Coulomb damped resonators that when operated at the resonant frequency, in the absence of parasitic damping and physical stops, the motion amplitude rises without limit unless the damping force satisfies a stability criterion [32]:

$$
F_{e} \geq \frac{\pi}{4} m \omega^{2} Y_{o}
$$

\section{4 - Example Operating Chart}

If we define $Z_{l} / Y_{0}$ and $\omega_{n}$ as our general operating parameters, then we can plot equations (2) and (11) as limits of operation for electromagnetic and piezoelectric devices. By adding the $Q_{p}$ of the device, and the combined $Q$, we can indicate regions of operation as a function of operating point.

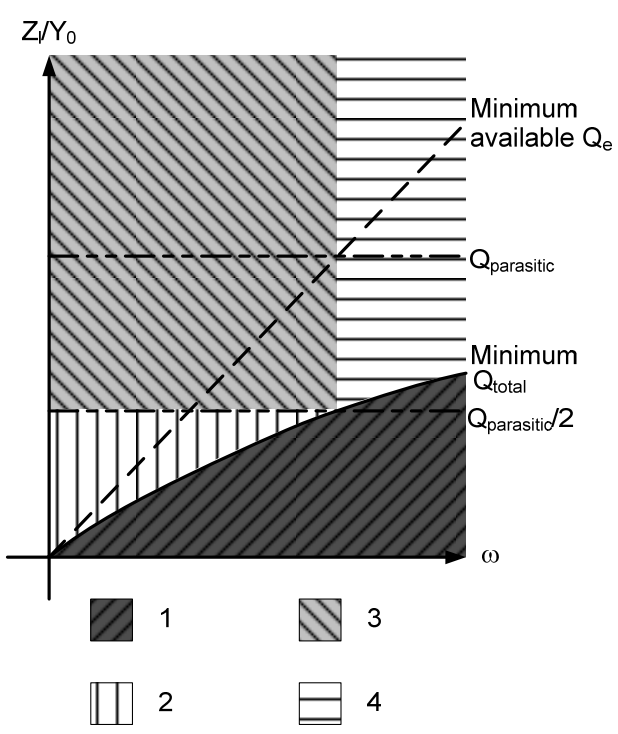

Figure 7 - Operating limits on electrical damping and parasitic Q factor.

This is done in Fig. 7 schematically, for an electromagnetic generator with parasitic damping present. The parasitic damping factor (and hence the $Q_{p}$ ) has been assumed to be constant with frequency for simplicity, but any actual frequency dependence could easily be substituted. For example, the parasitic damping force for a comb-drive as given by equation (14) is equivalent to a frequency independent damping coefficent $D$, and thus a $Q_{e}$ linearly proportional to frequency.

With reference to Fig. 7, The operating regions are as follows:

1. Harmonic motion is not possible. The maximum combined damping factor (which is $\left(1 / Q_{p}+1 / Q_{e}\right)$ ) is less than required for oscillation within the limit of $Z_{l}$. The line marked 'Minimum $\mathrm{Q}_{\text {total }}$ ' indicates the minimum value of $Z_{0} / Y_{0}$ achievable. If the operating point is below this line, harmonic motion is not possible and the mass will strike the end-stops.

2. Optimal operation occurs when the electrical damping and parasitic damping are equal, assuming that this results in $Z_{0}<Z_{l}$. In this operating region, if the parasitic and electrical damping terms were set equal, i.e. $Q_{e}=Q_{p}$, the mass would hit the end stops because the overall damping would not be large enough. Therefore, for optimal operation, the electrical damping should be set so that $Z_{0}=Z_{l}$, i.e. $Q_{e}>Q_{p}$. 
3. In this region of the figure, the parasitic and electrical damping terms should be set equal, i.e. $Q_{e}=Q_{p}$. The device will then operate within its displacement limit. This will give $Z_{0} / Y_{0}=Q_{\text {parasitic }} / 2$.

4. In this region it is not possible to make the electrical damping and parasitic damping equal, because the electrical damping force cannot be made large enough i.e. $Q_{e}>Q_{p}$. The electrical damping should therefore be set to the maximum that can be achieved. The generator can operate within the displacement constraint, but a different transducer could in principle extract more power if it could generate a higher damping force.

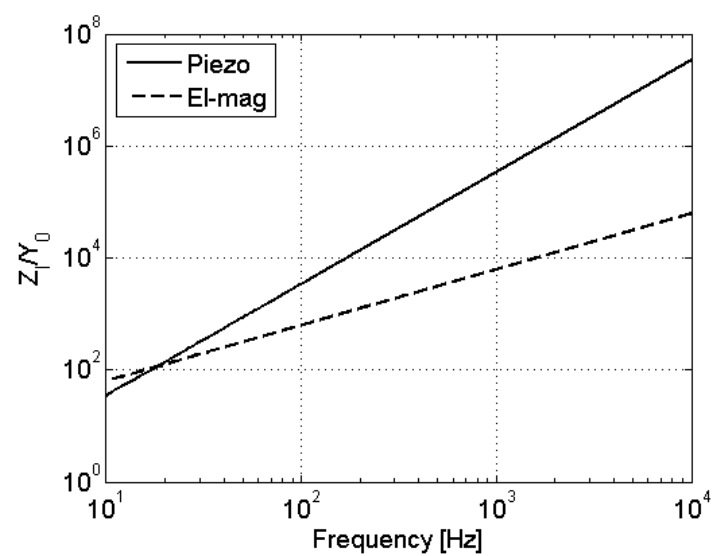

Figure 8 - Comparison of minimum Q factors with electromagnetic and piezoelectric cube devices of volume 1cc

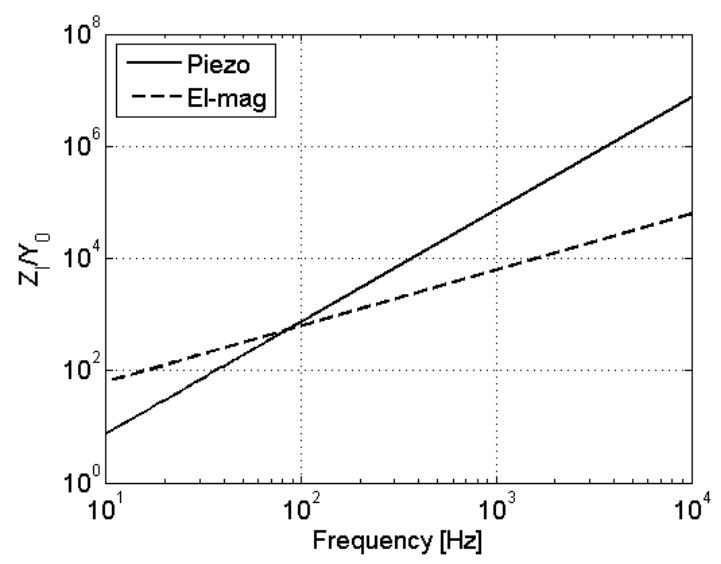

Figure 9 - Comparison of minimum Q factors with electromagnetic and piezoelectric cube devices of volume $0.1 \mathrm{cc}$.

Figures 8 and 9 show two specific examples of the minimum Q-factor achievable from electromagnetic, and piezoelectric generators. In each case we assume a cubic device of length $L$ and the mass, of relative density $8.9(\mathrm{Ni})$, is taken to occupy half the device volume. The electromagnetic device is assumed to have a flux density of 1 $\mathrm{T}$ and a copper coil occupying $2 \%$ of the device volume, for which a fixed $N^{2} / R_{i}$ is obtained. The active coil length $l$ is assumed to be $L / 2$. For the piezoelectric device, we assume $\varepsilon_{r}=1000$, area $L^{2}$, thickness $L / 10$ and $e_{33}=0.15 \mathrm{C} / \mathrm{m}^{2}$. A leverage factor of 500 was chosen. Because the $Q_{\min }$ for electromagnetic and piezoelectric devices scale as $\omega$ and $\omega^{2}$ respectively, there will always be a frequency above which electromagnetic devices can achieve higher damping. As can be seen in Figure 8 and Figure 9, the cross-over frequency increases as device size decreases. It can also be observed that the increasing Q with frequency (for both transducer types) could explain the decreasing performance trend seen in Fig. 4.

\section{3 - CONCLUSIONS}

Obtaining maximum power from inertial energy scavengers is often limited by the maximum damping force achievable in the transduction mechanism, and this problem increases with increasing frequency. Piezoelectric generators can outperform electromagnetic generators at low frequency, but with increasing frequency, the internal capacitance of the piezoelectric reduces the amount of real power that can be obtained. This suggests that piezoelectric devices might be better suited to human body powered applications and electromagnetic devices to high frequency applications.

\section{REFERENCES}

[1] S. Roundy, P. K. Wright, and J. M. Rabaey, Energy Scavenging for Wireless Sensor Networks. Boston, Massachusetts: Kluwer Academic Publishers, 2003. J. A. Paradiso and T. Starner, "Energy scavenging for mobile and wireless electronics," Pervasive Computing, IEEE, vol. 4, pp. 18-27, 2005.

[3] P. D. Mitcheson, T. C. Green, E. M. Yeatman, and A. S. Holmes, "Architectures for vibration-driven micropower generators," Microelectromechanical Systems, Journal of, vol. 13, pp. 429-440, 2004.

[4] C. Shearwood and R. B. Yates, "Development of an electromagnetic microgenerator," Electronics Letters, vol. 33, pp. 1883-1884, 1997.

[5] C. B. Williams, C. Shearwood, M. A. Harradine, P. H. Mellor, T. S. Birch, and R. B. Yates, "Development of an electromagnetic micro-generator," Circuits, Devices and Systems, IEE Proceedings, vol. 148, pp. 337-342, 2001. W. J. Li, G. M. H. Chan, N. N. H. Ching, P. H. W. Leong, and H. Y. Wong, "Dynamical modelling and Simulation of a Laser-Micromachined Vibration-Based Micro Power Generator," International Journal of Nonlinear Sciences and Simulation, vol. 1, pp. 345-353, 2000.

[7] W. J. Li, T. C. H. Ho, G. M. H. Chan, P. H. W. Leong, and H. Y. Wong, "Infrared signal transmission by a lasermicromachined, vibration-induced power generator," presented at Circuits and Systems, 2000. Proceedings of the 43rd IEEE Midwest Symposium on, 2000.

[8] W. J. Li, Z. Wen, P. K. Wong, G. M. H. Chan, and P. H. W. Leong, "A Micromachined Vibration-Induced Power Generator for Low Power Sensors or Robotic Systems," presented at 8th International Symposium on Robotics with Applications, Hawaii, 2000.

[9] N. N. H. Ching, G. M. H. Chan, W. J. Li, H. Y. Wong, and Z. Wen, "A laser-micromachined Vibrational to Electrical Power Transducer for Wireless Sensing Systems," presented at 11th International Conference on Solid-State Sensors and Actuators, Munich, Germany, 2000 . 
[10] N. Ching, "A laser-micromachined multi-modal resonating power transducer for wireless sensing systems," Sensors and actuators. A, Physical, vol. 97, pp. 685, 2002.

[11] N. N. H. Ching, G. M. H. Chan, W. J. Li, H. Y. Wong, and P. H. W. Leong, "PCB Integrated Micro-Generator for Wireless Systems," presented at International Symposium on Smart Structures, Hong Kong, 2000.

[12] J. M. H. Lee, S. C. Yuen, W. J. Li, and P. H. W. Leong, "Development of an AA Size Energy Transducer with Micro-Resonators," presented at International Symposium on Circuits and Systems, 2003.

[13] M. El-hami, P. Glynne-Jones, N. M. White, M. Hill, S. Beeby, E. James, A. D. Brown, and J. N. Ross, "Design and fabrication of a new vibration-based electromechanical power generator," Sensors and Actuators A: Physical, vol. 92, pp. 335-342, 2001.

[14] P. Glynne-Jones, M. J. Tudor, S. P. Beeby, and N. M. White, "An electromagnetic, vibration-powered generator for intelligent sensor systems," Sensors and Actuators A: Physical, vol. 110, pp. 344-349, 2004.

[15] H. Kulah and K. Najafi, "An electromagnetic micro power generator for low-frequency environmental vibrations," presented at Micro Electro Mechanical Systems, 2004. 17th IEEE International Conference on. (MEMS), 2004.

[16] M. Mizuno and D. G. Chetwynd, "Investigation of a resonance microgenerator," Journal of Micromechanics and Microengineering, vol. 13, pp. 209-216, 2003.

[17] M. Miyazaki, H. Tanaka, G. Ono, T. Nagano, N. Ohkubo, and T. Kawahara, "Electric-Energy Generation Using Variable-Capacitive Resonator for Power-Free-LSI," IEICE Transactions on Electronics, vol. E87-C, pp. 549$555,2004$.

[18] M. Miyazaki, H. Tanaka, G. Ono, T. Nagano, N. Ohkubo, T. Kawahara, and K. Yano, "Electric-Energy Generation Using Variable-Capacitance Resonator for Power-Free LSI:Efficiency Analysis and Fundamental Experiment," presented at International Symposium on Low Power Electronics and Design, 2003.

[19] P. D. Mitcheson, P. Miao, B. H. Stark, E. M. Yeatman, A. S. Holmes, and T. C. Green, "MEMS electrostatic micropower generator for low frequency operation," Sensors and Actuators A: Physical, vol. 115, pp. 523-529, 2004.

[20] P. Glynne-Jones, M. El-hami, S. Beeby, E. James, A. D. Brown, M. Hill, and N. M. White, "A Vibration-Powered Generator for Wireless Microsystems," presented at International Symposium on Smart Structures and Microsystems, 2000.

[21] P. Glynne-Jones, S. P. Beeby, and N. M. White, "Towards a piezoelectric vibration-powered microgenerator," Iee Proceedings-Science Measurement and Technology, vol. 148, pp. 68-72, 2001.

[22] N. M. White, P. Glynne-Jones, and S. P. Beeby, "A novel thick-film piezoelectric micro-generator," Smart Materials \& Structures, vol. 10, pp. 850-852, 2001.

[23] P. Glynne-Jones, S. Beeby, E. James, and N. M. White, "The modelling of a piezoelectric vibration powered generator for microsystems," presented at Transducers '01, Eurosensors XV, Munich, Germany, 2001.

[24] E. Koukharenko, S. P. Beeby, M. J. Tudor, N. M. White, T. O'Donnell, C. Saha, S. Kulkarni, and S. Roy, "Microelectromechanical systems vibration powered electromagnetic generator for wireless sensor applications," Microsystem Technologies-Micro-and Nanosystems-Information Storage and Processing Systems, vol. 12, pp. 1071-1077, 2006.
[25] M. Duffy and D. Carroll, "Electromagnetic generators for power harvesting," presented at Power Electronics Specialists Conference, 2004. PESC 04. 2004 IEEE 35th Annual, 2004.

[26] K. Hammond, E. S. Leland, J. Baker, E. Carleton, E. Reilly, E. Lai, B. Otis, J. M. Rabaey, V. Sundararajan, and P. K. Wright, "An Integrated Node for EnergyScavenging, Sensing and Data Transmission: Applications in Medical Diagnostics," presented at 2nd International Workshop on Wearable and Implantable Body Sensor Networks, Imperial College London, 2005.

[27] N. Ching, "A laser-micromachined multi-modal resonating power transducer for wireless sensing systems," presented at 11th International Conference on Solid State Sensors and Actuators, Munich, Germany, 2001.

[28] P. Miao, P. D. Mitcheson, A. S. Holmes, E. M. Yeatman, T. C. Green, and B. H. Stark, "Mems inertial power generators for biomedical applications," Microsystem Technologies-Micro-and Nanosystems-Information Storage and Processing Systems, vol. 12, pp. 1079-1083, 2006.

[29] E. K. Reilly, E. Carleton, and P. K. Wright, "Thin film piezoelectric energy scavenging systems for long term medical monitoring," presented at Wearable and Implantable Body Sensor Networks, 2006. BSN 2006. International Workshop on, 2006.

[30] D. Guyomar, A. Badel, E. Lefeuvre, and C. Richard, "Toward energy harvesting using active materials and conversion improvement by nonlinear processing," IEEE Transactions on Ultrasonics Ferroelectrics and Frequency Control, vol. 52, pp. 584-595, 2005.

[31] B. Borovic, F. L. Lewis, A. Q. Liu, E. S. Kolesar, and D. Popa, "The lateral instability problem in electrostatic comb drive actuators: modeling and feedback control," Journal of Micromechanics and Microengineering, vol. 16, pp. 1233, 2006.

[32] J. P. Den Hartog, Mechanical Vibrations. Mineola, NY: Dover Publications, 1985. 